\title{
Metabolic health, menopause, and physical activity-a 4-year follow-up study
}

\author{
Matti Hyvärinen $\mathbb{I D}^{1,6 \times}$, Hanna-Kaarina Juppi $\mathbb{D}^{1,6}$, Sara Taskinen $\mathbb{I D}^{2}$, Jari E. Karppinen $\mathbb{I D}^{3}$, Sira Karvinen ${ }^{1}$, Tuija H. Tammelin ${ }^{4}$, \\ Vuokko Kovanen (D) ${ }^{3}$, Pauliina Aukee ${ }^{5}$, Urho M. Kujala (D) ${ }^{3}$, Timo Rantalainen (D) ${ }^{1}$, Sarianna Sipilä (D) ${ }^{1}$ and Eija K. Laakkonen (D) ${ }^{1}$
}

(c) The Author(s) 2021

BACKGROUND: In women, metabolic health deteriorates after menopause, and the role of physical activity (PA) in mitigating the change is not completely understood. This study investigates the changes in indicators of metabolic health around menopause and evaluates whether PA modulates these changes.

METHODS: Longitudinal data of 298 women aged $48-55$ years at baseline participating in the ERMA and EsmiRs studies was used. Mean follow-up time was 3.8 (SD 0.1) years. Studied indicators of metabolic health were total and android fat mass, waist circumference, waist-to-hip ratio (WHR), systolic (SBP) and diastolic (DBP) blood pressure, blood glucose, triglycerides, serum total cholesterol, and high- (HDL-C) and low-density (LDL-C) lipoprotein cholesterol. PA was assessed by accelerometers and questionnaires. The participants were categorized into three menopausal groups: PRE-PRE (pre- or perimenopausal at both timepoints, $n=56$ ), PRE-POST (pre- or perimenopausal at baseline, postmenopausal at follow-up, $n=149$ ), and POST-POST (postmenopausal at both timepoints, $n=93$ ). Analyses were carried out using linear and Poisson mixed-effect models.

RESULTS: At baseline, PA associated directly with HDL-C and inversely with LDL-C and all body adiposity variables. An increase was observed in total $(B=1.72,95 \% \mathrm{Cl}[0.16,3.28])$ and android fat mass $(0.26,[0.06,0.46]), \operatorname{SBP}(9.37,[3.34,15.39])$, and in all bloodbased biomarkers in the PRE-POST group during the follow-up. The increase tended to be smaller in the PRE-PRE and POST-POST groups compared to the PRE-POST group, except for SBP. The change in PA associated inversely with the change in SBP (-2.40, $[-4.34,-0.46])$ and directly with the change in WHR $(0.72,[0.05,1.38])$.

CONCLUSIONS: In middle-aged women, menopause may accelerate the changes in multiple indicators of metabolic health. PA associates with healthier blood lipid profile and body composition in middle-aged women but does not seem to modulate the changes in most of the studied metabolic health indicators during the menopausal transition.

International Journal of Obesity (2022) 46:544-554; https://doi.org/10.1038/s41366-021-01022-x

\section{INTRODUCTION}

Metabolic health is an umbrella term for factors that combine several aspects of cellular, cardiovascular, and cardiorespiratory health and well-being. Body adiposity, anthropometrics, blood pressure, and blood-based biomarkers, such as serum lipids and blood glucose, can be clinically used to evaluate metabolic health. One established method is to use the diagnostic criteria of metabolic syndrome (MetS) [1], a multifaceted disorder predisposing individuals to severe health concerns, such as atherosclerotic heart disease [2] and type II diabetes [3]. Although there is a significant genetic component in the individual variance of metabolic health and emergence of MetS risk factors [4], unhealthy lifestyle habits, such as physical inactivity, are proposed to be a major contributor.

The effect of menopause on metabolic health and the development of MetS has been an increasing area of interest, as nowadays women in Western countries are expected to live in the postmenopausal state for more than one third of their lives [5-7]. Menopausal transition and the accompanying changes in the hormonal milieu (e.g., decrease in the systemic estradiol (E2) levels) have been associated with unfavorable changes in several indicators of metabolic health $[8,9]$. For instance, increased blood glucose [10], accumulation of abdominal adiposity [11] as well as unhealthy changes in serum lipids [12] have been reported during menopausal transition. Additionally, menopause-related increase in inflammation marker levels [13] and decrease in muscle mass [14] have an additive negative impact on metabolic health. Therefore, it is not surprising that in women the incidence of MetS and cardiovascular disease increases after menopause $[8,15]$.

Physical activity (PA) has been widely proposed to improve the metabolic risk factor profile and cardiovascular health. Literature suggests that regular PA decreases total and visceral fat mass,

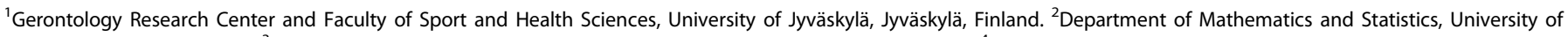

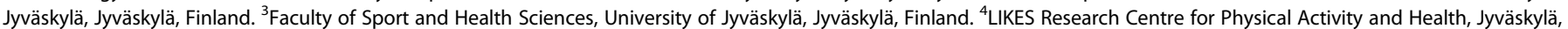

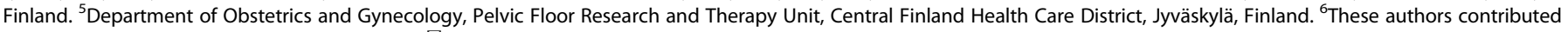
equally: Hyvärinen Matti, Juppi Hanna-Kaarina. ${ }^{\circledR}$ email: matti.v.hyvarinen@jyu.fi 
improves insulin sensitivity, prevents dyslipidemia, and decreases systolic (SBP) and diastolic (DBP) blood pressure [16-18]. Thus, it can be used for the prevention and treatment of MetS. However, the associations between PA and changes in indicators of metabolic health around menopause are understudied as only few longitudinal studies have been conducted using devicemeasured PA [12, 19]. Moreover, these studies included only women transitioning from pre- or perimenopause to postmenopause and therefore could not address the contemporaneous aging-related changes.

The objective of this study was to investigate the changes around menopause in serum lipids and glucose, blood pressure, and body adiposity as indicators of metabolic health. Additionally, the aim was to evaluate whether PA modulates these changes using unique longitudinal data from the study of middle-aged women with different menopausal status.

\section{MATERIALS AND METHODS}

\section{Study design and population}

This study utilized the data from the observational Estrogenic Regulation of Muscle Apoptosis (ERMA) and Estrogen, MicroRNAs and the Risk of Metabolic Dysfunction (EsmiRs) studies. The participant selection for the ERMA study has been described in detail elsewhere [20]. Briefly, out of the 6878 randomly selected women aged 47-55 years living in Central Finland, 1393 consented and met the inclusion criteria for the baseline measurements (Fig. 1). Exclusion criteria included conditions and the use of medications affecting ovarian function and systemic hormone or inflammatory status, such as bilateral oophorectomy, pregnancy, lactating, severe obesity (self-reported body mass index $(\mathrm{BMI}) \geq 35 \mathrm{~kg} / \mathrm{m}^{2}$ ), or the use of estrogen-containing medications and continuous cortisone or inflammatory drug treatment [20].

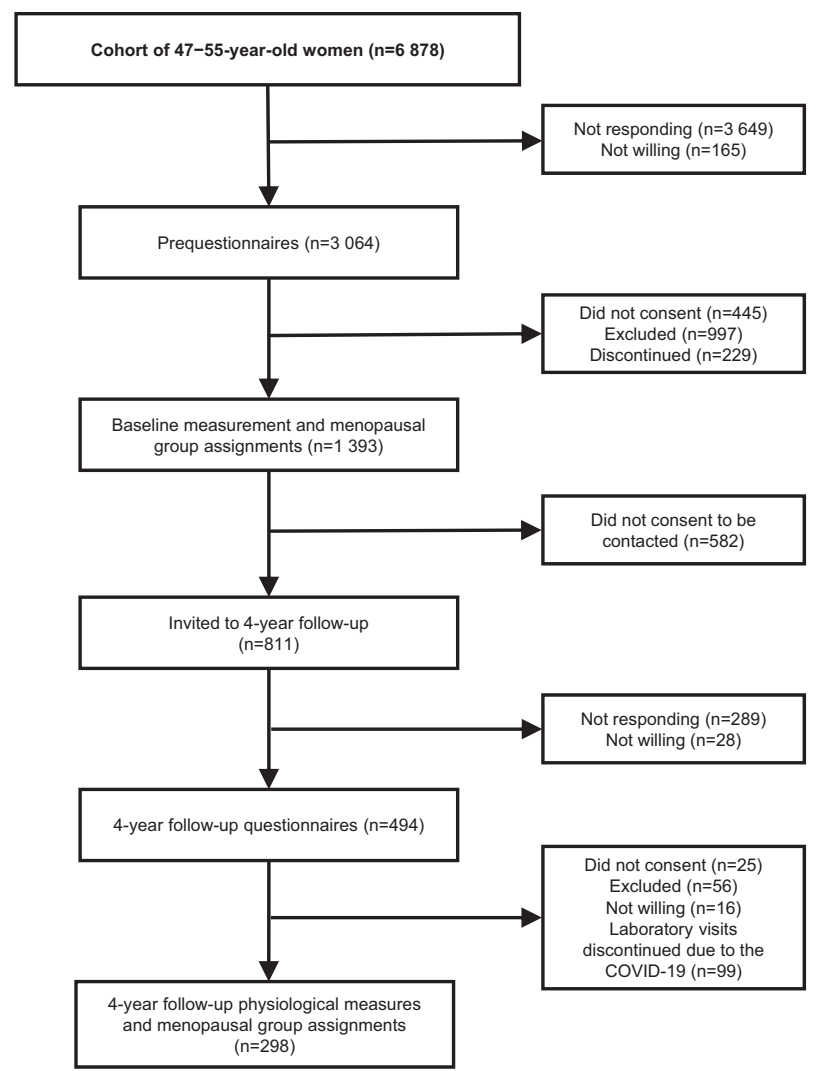

Fig. 1 Flow chart of the study. The flow chart describes the participant enrollment and selection procedure of the ERMA and EsmiRs studies with detailed information about the exclusions and discontinuations during each phase of the study.
The 4-year follow-up measurements were carried out in the EsmiRs study. Out of the 811 participants measured in the ERMA baseline who consented to be contacted, 494 were willing to participate in the EsmiRs questionnaire. Of these participants, 56 were excluded, 25 did not consent, and 16 were not willing to continue to physiological measurements. The participants were excluded due to having more than 7 years from menopause based on the self-reports $(n=46)$, diabetes requiring regular insulin therapy $(n=2)$, severe cardiovascular dysfunction $(n=2)$, or diagnosed with cancer during the follow-up $(n=6)$. Furthermore, 99 participants could not be measured because of the COVID-19 lockdown. Consequently, the final study sample included 298 white women (Fig. 1). To estimate potential selection bias, sensitivity analyses comparing the included sample to the rest of the measured participants $(n=1095)$ at the ERMA baseline for all outcome variables and accelerometer-measured PA were conducted.

The recruiting for the ERMA study was conducted in 2014. The baseline measurements were initiated at the beginning of 2015, and they lasted until the end of 2016. The recruiting for the EsmiRs study started in November 2018 and laboratory measurements were initiated in January 2019. They were discontinued on March 16, 2020 due to the COVID-19 pandemic. The study was performed in accordance with the Declaration of Helsinki. All participants provided written informed consent, and the study was approved by the ethical committee of the Central Finland Health Care District (ERMA 8U/2014 and EsmiRs 9U/2018).

\section{Menopausal status assignments}

Blood sampling after overnight fasting was performed in a supine position from the antecubital vein during days 1-5 of menstrual cycle if the cycle was predictable. Serum was separated from whole blood and stored at $-80^{\circ} \mathrm{C}$ before analysis. Serum concentrations of E2 and follicle-stimulating hormone (FSH) were determined using IMMULITE 2000 XPi (Siemens Healthineers, Erlangen, Germany) according to the manufacturer's instructions.

Participants were categorized as pre-, peri-, or postmenopausal in both measurements based on the FSH concentrations and self-reported menstrual bleeding diaries using the adapted Stages of Reproductive Aging Workshop (STRAW + 10) guidelines [20]. The participants were divided into three groups based on how their menopausal status changed during the study. PRE-POST group $(n=149)$ consisted of women who experienced menopause during the follow-up period. That is, they were categorized as pre- or perimenopausal in the baseline and postmenopausal in the follow-up measurement. Furthermore, women that were pre- or perimenopausal (PRE-PRE, $n=56$ ) or postmenopausal (POST-POST, $n=93$ ) in both measurements were designated to their respective groups.

\section{Indicators of metabolic health}

Blood pressure and anthropometrics were measured after overnight fasting. SBP and DBP was measured twice in a sitting position after $10 \mathrm{~min}$ rest using Omron M6 Comfort (Omron Healthcare, Kioto, Japan) with a standard size cuff and the mean values of the measurements were used. Waist circumference was measured in light underwear midway between the superior iliac spine and the lower rib margin, and hip circumference at the level of the greater trochanters [21]. Body mass and height were measured with standard procedures and BMI was computed by dividing the body mass with squared body height. Total body fat mass and percentage, android fat mass, and fat free mass were assessed with dualenergy X-ray absorptiometry (DXA; LUNAR, GE Healthcare, Chicago, IL, USA).

Serum samples collected during menopausal status assignment were also used for outcome variable analysis. Serum glucose, high- (HDL-C), lowdensity lipoprotein cholesterol (LDL-C), total cholesterol, and triglycerides were measured with KONELAB 20 XTi analyzer (Thermo Fischer Scientific, Vantaa, Finland).

The updated ATP III criteria for MetS risk factors was used [1]. The defining levels for risk factors were $\geq 88 \mathrm{~cm}$ for waist circumference, $\geq 130$ / $\geq 85 \mathrm{mmHg}$ for blood pressure, $\geq 1.69 \mathrm{mmol} / \mathrm{l}$ for serum triglycerides, $\geq 5.6 \mathrm{mmol} / \mathrm{l}$ for blood glucose, and $<1.29 \mathrm{mmol} / \mathrm{l}$ for HDL-C.

\section{Physical activity}

Accelerometry-measured PA was assessed in both timepoints by triaxial ActiGraph GT3X and wGT3X accelerometers (ActiGraph LLC, Pensacola, FL, USA) with an accompanied diary. Participants were instructed to wear the accelerometers for seven consecutive days on their right hip during 
waking hours, except during water-based activities. The data were collected at $60 \mathrm{~Hz}$ and the Euclidian norm of the resultant acceleration was computed for each timepoint. Consequently, the mean amplitude deviations (MAD) were computed for non-overlapping $5 \mathrm{~s}$ epochs, and the mean MAD value for 1 min epochs were determined based on the $5 \mathrm{~s}$ MAD values [22]. The accelerometer-measured MAD (ACC-MAD) reflects the directly measured acceleration and captures the volume of the activity in the entire intensity profile [23] and has been validated against oxygen consumption [24]. Non-wear time was identified as any epoch of at least 60 min with 1 min MAD continuously less than $0.001 \mathrm{~g}$ (g denotes the gravitational acceleration on Earth). ${ }^{1} \mathrm{~A}$ minimum of 3 days with a wear time of $10 \mathrm{~h}$ or more was regarded as a valid measurement. ${ }^{2}$ Finally, the ACC-MAD was determined for wear time for each measurement. For supplementary information, we defined activity with intensity higher or equal to $0.091 \mathrm{~g}$ as moderate-to-vigorous physical activity (MVPA) [24]. The ACC-MAD was strongly associated $(r=0.88$ and $r=0.79)$ with the amount of MVPA and ActiGraph counts [25], respectively.

Additionally, PA was assessed by a self-reported questionnaire (SR-PA) [26]. Briefly, the questionnaire included four questions about the average frequency, intensity, and duration of leisure time PA bouts as well as the average duration of the commuting activity. Based on the responses, the metabolic equivalent (MET) hours per day for leisure time PA was calculated.

\section{Covariates}

The use of medications and lifestyle habits were assessed by a structured questionnaire at baseline and follow-up measurements. Responses were used to assess alcohol consumption in portions per week and current smoking status (nonsmoker/smoker). Participants also reported their use of regular prescription medications that were categorized using the Anatomical Therapeutic Chemical (ATC) classification [27]. The use of medications was assessed (non-user/user) separately in preparations affecting blood pressure (ATC C02-05 and C07-09), serum lipids (ATC C10), and thyroid function (ATC H03).

Based on self-reports, participants were classified as being either nonuser, only estrogen, only progestogen or combined estrogen and progestogen users. Exogenous sex hormone preparations for contraceptive and hormone replacement therapy use, such as pills, intra-uterine device, patches, and transdermal gels but not intravaginal local estrogen therapy were included. Diet quality score (DQS) was computed based on a food-frequency questionnaire as reported previously [14]. Shortly, DQS consisted of 11 elements characteristic to a healthy diet by the Nordic Nutrition Recommendations 2012. A higher intake of whole-grains, vegetables, fruits and berries, low-fat dairy, fish, and nuts and seeds, and a lower intake of processed grains, processed meats, sugary beverages, fast foods, and sweet or salty snacks were regarded as beneficial. Each component accounted for was worth of 1 point, and the maximum score available was therefore 11 points. A higher DQS score was regarded to reflect a healthier diet. The DQS was partly adapted from Masip et al. [28].

\section{Missing data}

The percentage of missing values across the variables separately for each timepoint varied from 0 to $21 \%$. The number of valid measurements for 298 participants in each variable is presented in Table 1. The total number of missing data values was 741 out of 13,708 (5\%). Missing data occurred due to invalid or missing measurements as well as unclear or incomplete questionnaire responses. Missing data were assumed to occur at random and multiple imputation was used to create and analyse 50 multiply imputed data sets. Multiple imputation was carried out in R [29] using the "mice" package [30]. All variables measured at the same timepoint and the target variable measurement from the other timepoint were used for imputation of each variable. The number of iterations was set to 50 and passive imputation was used for the derived waist-to-hip ratio (WHR) variable. The model parameters were estimated in each imputed dataset separately and pooled using Rubin's rules [31]. For comparison, we also

\footnotetext{
${ }^{1}$ The threshold of $0.001 \mathrm{~g}$ was determined based on the correspondence with the self-reported wear time in this population $(r=0.70)$. Self-reported wear time was not used in the analysis due to the invalid and missing entries in the diaries.

${ }^{2}$ Seven valid days were recorded in $89 \%(462 / 528)$ of the measurements.
}

performed the complete case analysis and there was no notable difference in the results that would have led to different conclusions.

\section{Statistical analysis}

The main analyses were carried out using linear and Poisson mixed-effect models with random intercept [32]. For each outcome variable, the fixed effects were time $(0=$ baseline, $1=$ follow-up), menopausal group, ACC$M A D$, and interactions between time and group as well as time and ACCMAD. The interactions were included in the models to study how the change in PA associate with the change in outcome variables during the follow-up. Furthermore, the covariates included as fixed effects were mean centered age at baseline and the use of hormonal preparations. Residual plots, Q-Q plots, and correlation analysis were used for testing the model assumptions. The analyses were carried out in R using the "nIme" [33] and "Ime4" [34] packages.

Based on the literature, we identified candidate covariates related to lifestyle habits and the use of medications that may be associated with the outcome variables. Their distributions in the study population are presented in detail in Supplementary Table 1. However, to our consideration, lifestyle habits and the use of antihypertensives, lipidmodifying agents or thyroid therapy do not significantly affect the progression of menopausal transition, and the use may even be caused by the menopause-induced changes in the outcome variables. Thus, only the use of sex hormone therapy was controlled for confounding. Nonetheless, we also performed the analysis including the relevant variables and their interaction with time as covariates, but it did not have a notable effect on the results. Furthermore, we conducted sensitivity analyses for blood lipids and blood pressure by excluding the participants who used lipid-modifying agents and antihypertensives, respectively.

\section{RESULTS}

\section{Characteristics of the study population}

The average follow-up-time was 3.8 years in all groups (Table 1). At baseline, the participants were slightly overweight with mean BMI of $25.3 \pm$ SD 3.7 and had slightly elevated SBP $(132.0 \pm 3.7)$, DBP $(84.1 \pm 9.2)$, total cholesterol $(5.23 \pm 0.91)$, and LDL-C $(3.05 \pm 0.80)$. Other outcome variable means were within the normal range $[35,36]$. Participants in the PRE-PRE group were the youngest and had the lowest FSH and highest E2 levels at baseline. Respectively, the participants in the POST-POST group were the oldest and had the highest FSH and lowest E2 levels. The most notable changes in E2 and FSH levels occurred in the PRE-POST group during the follow-up. The percentage of the participants with three or more MetS risk factors was $16 \%$ at baseline and at follow-up. The sensitivity analyses using unpaired $T$-test indicated the study sample to have slightly lower blood glucose $(5.15 \pm 0.45$ and $5.28 \pm 0.63, t(1387)=3.319, p=0.001)$ and higher ACC-MAD $(30.2 \pm 10.0$ and $28.8 \pm 8.8, t(782)=-2.044, p=0.041)$ compared to participants that did not participate in the follow-up. No differences were observed for other outcome variables (data not shown).

\section{Blood-based biomarkers}

The PRE-POST group had lower total cholesterol and HDL-C compared to the POST-POST group (Table 2). In the full sample, ACC-MAD was directly associated with HDL-C $(B=0.06,95 \% \mathrm{Cl}$ $[0.01,0.11])$ and inversely with $\operatorname{LDL}-\mathrm{C}(B=-0.11,95 \% \mathrm{Cl}[-0.21$, $-0.01])$. The levels of all blood-based biomarkers increased during the follow-up in the PRE-POST group and the increase tended to be smaller in the PRE-PRE and, especially, in the POSTPOST group. The change in ACC-MAD was not associated with the change in any of the outcome variables measured from blood. The use of progestogen was associated with lower HDL-C, while the combined progestogen and estrogen use was associated with a lower blood glucose. The results did not differ notably when using SR-PA as a PA measure (Supplementary Table 2) or excluding participants using lipid-modifying agents (Supplementary Table 3). 


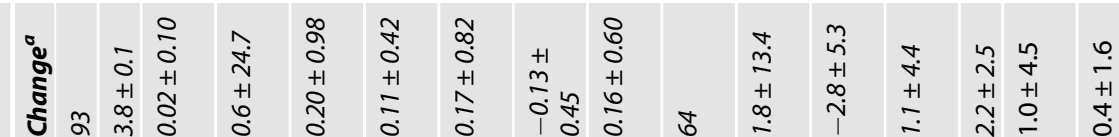
ঊ

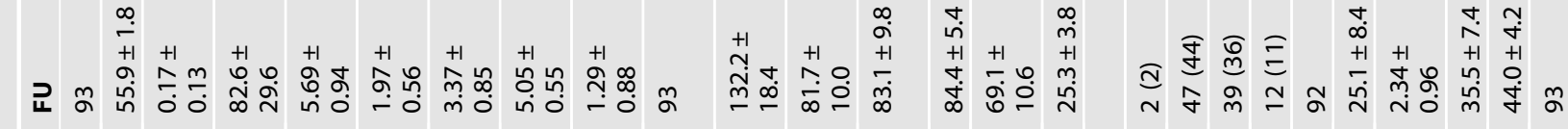
苔

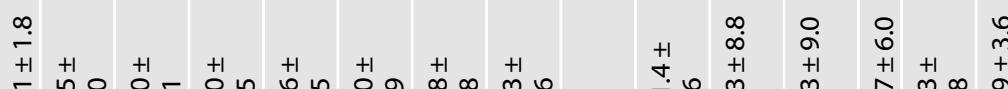

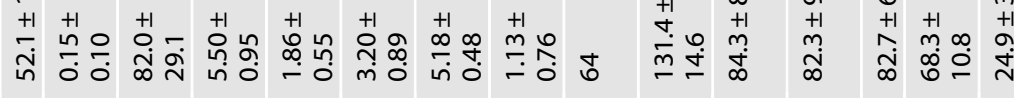

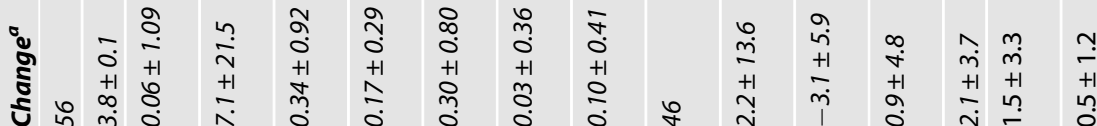

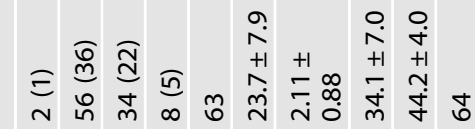

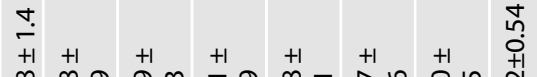
วน

\section{告}

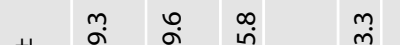

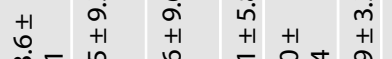

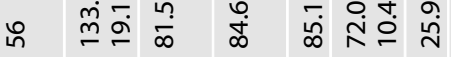

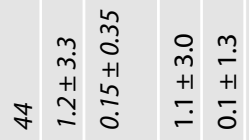

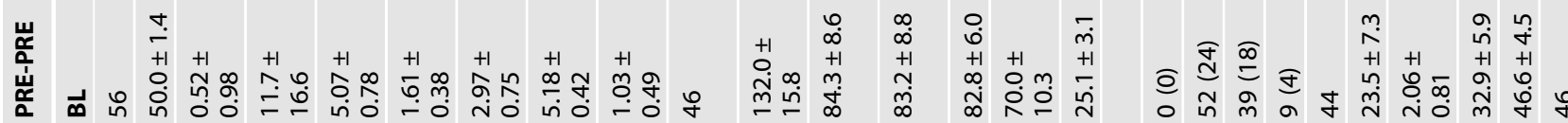

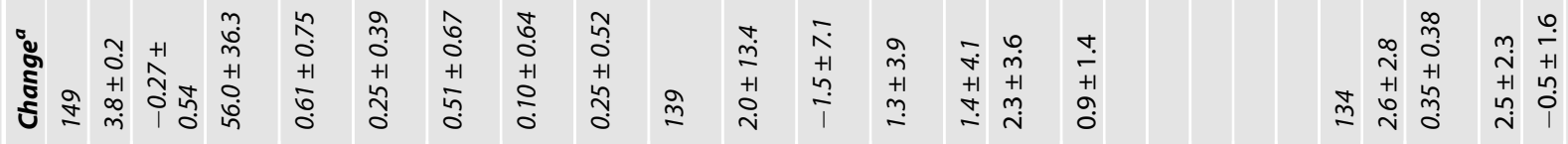

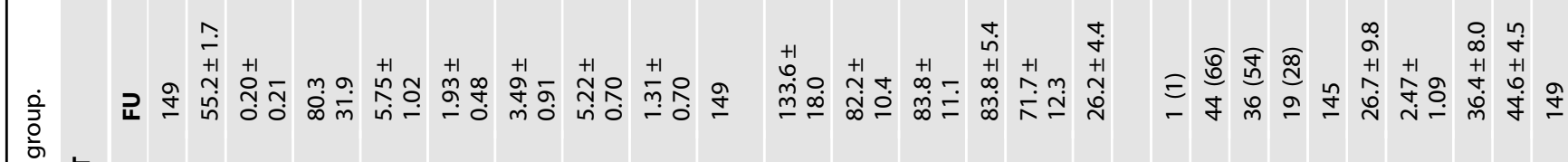

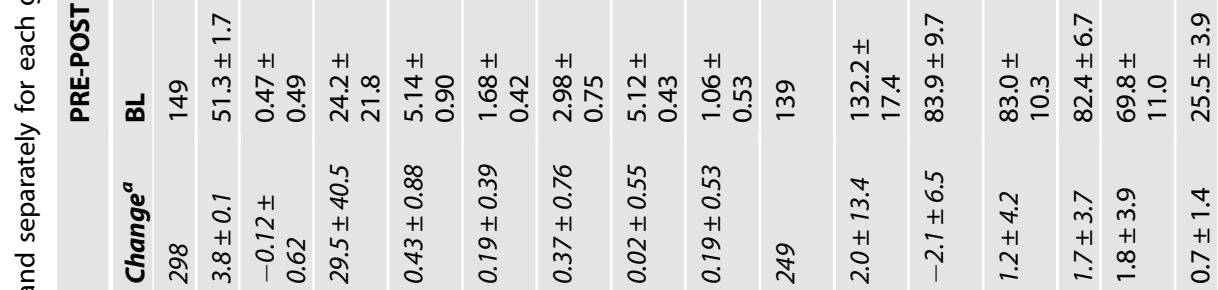

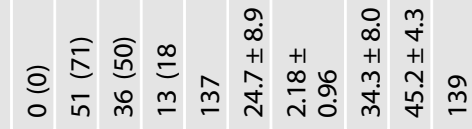

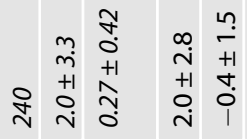

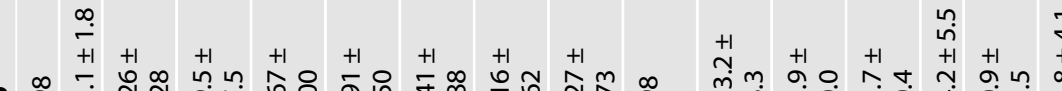

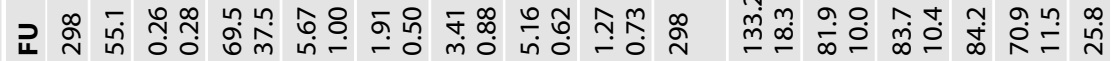

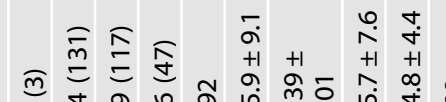

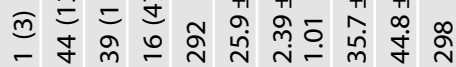

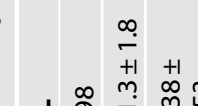

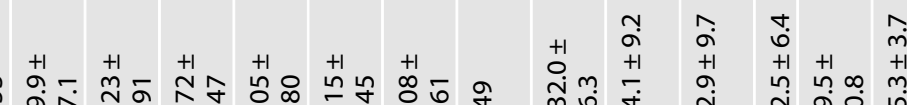
$\stackrel{\substack{m \\+1 \\ m}}{\stackrel{n}{n}}$

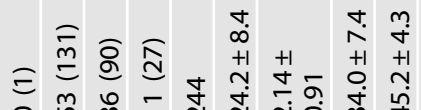

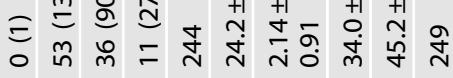
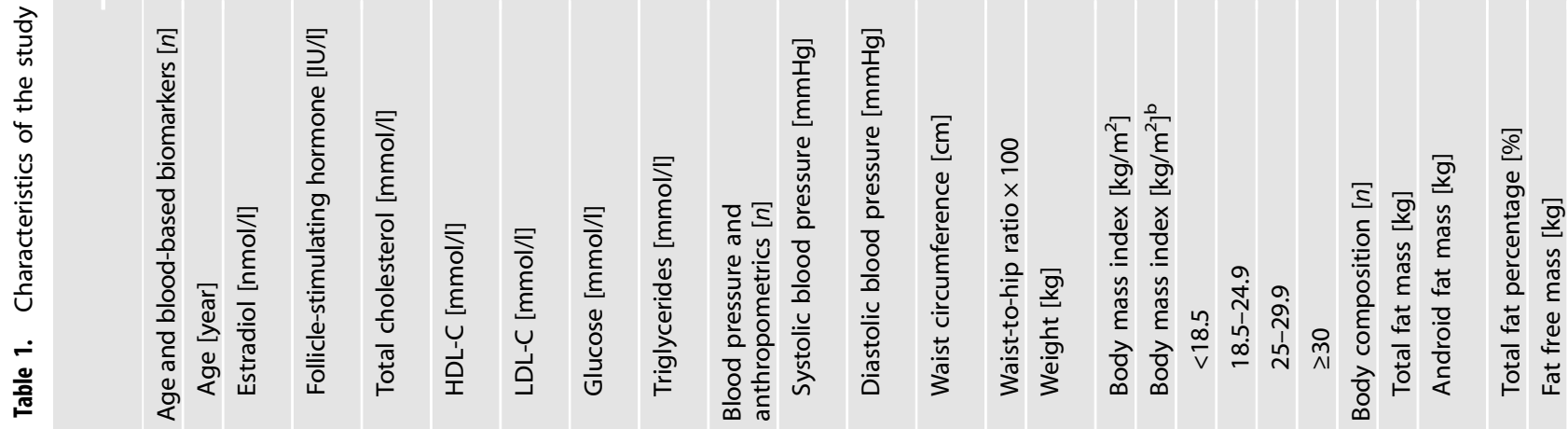


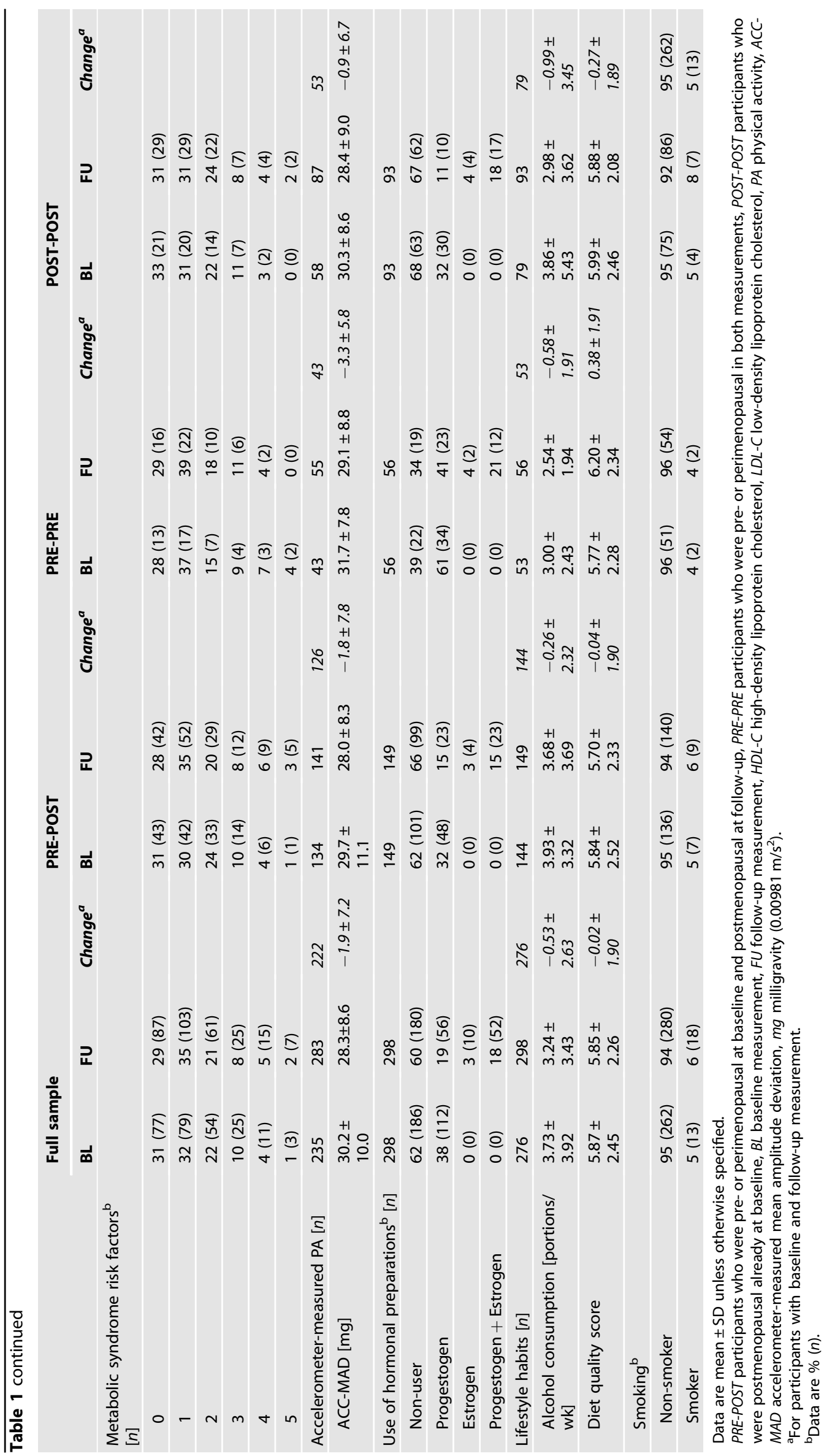




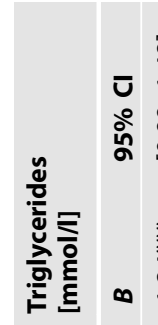

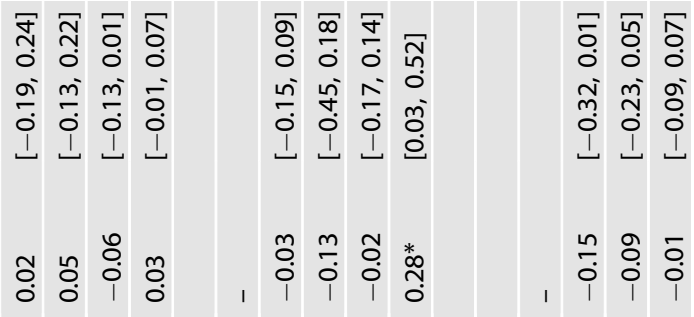

v
in
in
ஸें
ò

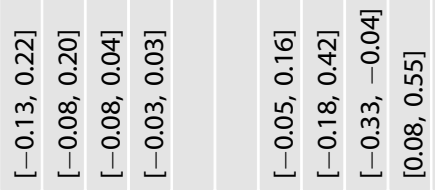

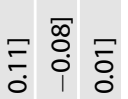

กิ

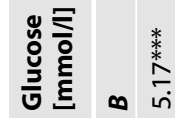

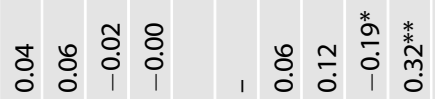

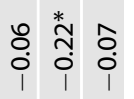

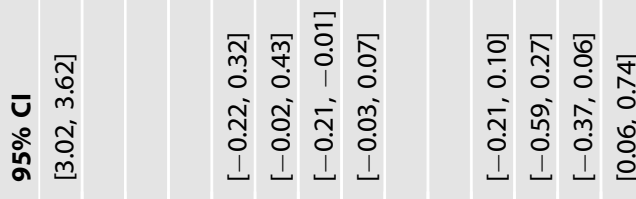

సี.

卞

i 1 i

光完

Uి

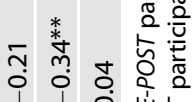

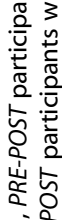

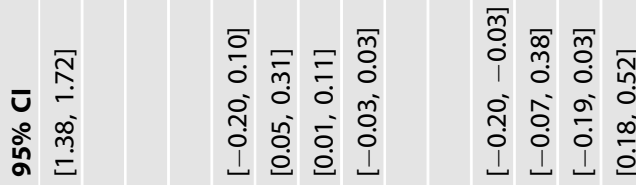

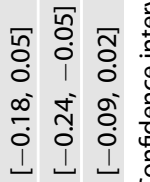

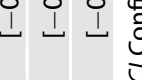

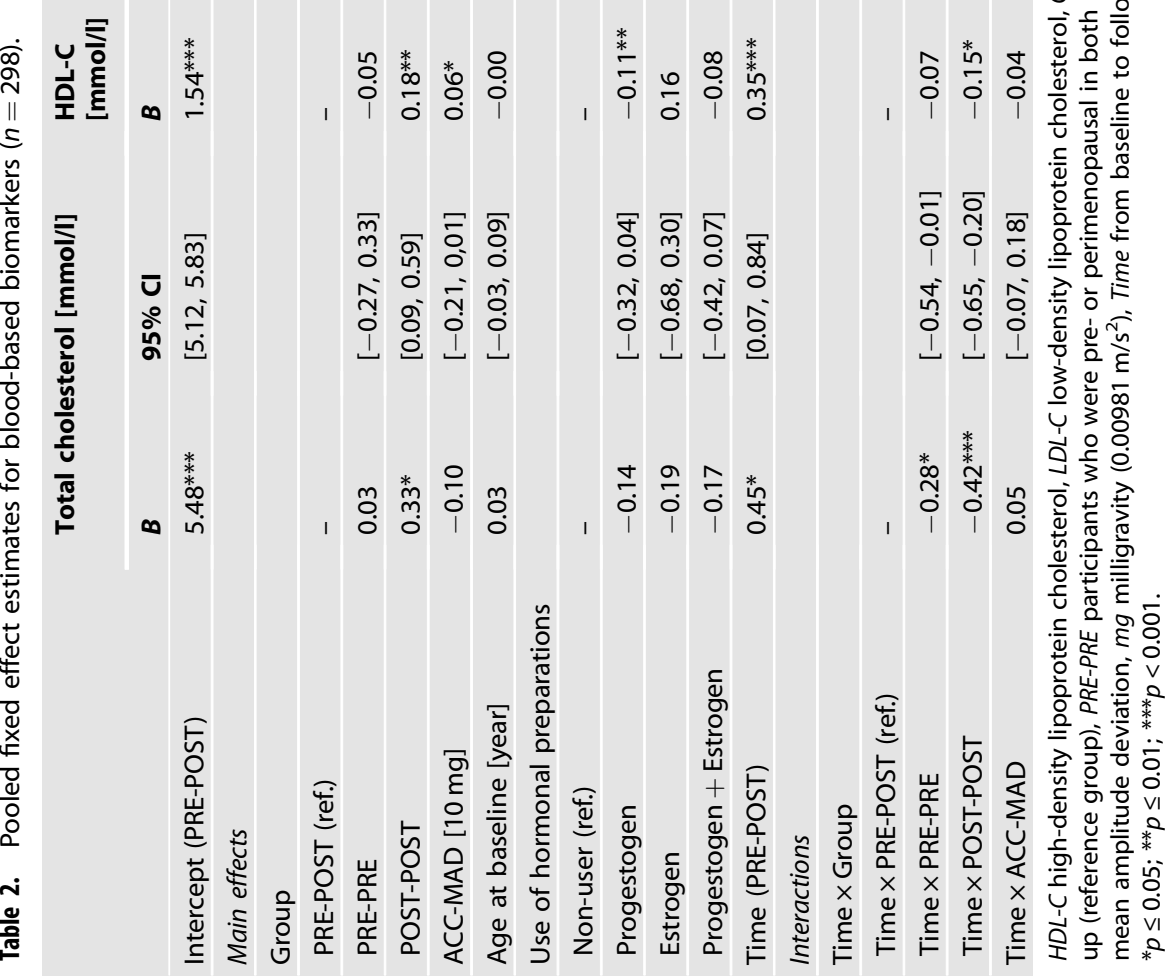


Table 3. Pooled fixed effect estimates for body composition and anthropometrics ( $n=298)$.

\begin{tabular}{|c|c|c|c|c|c|c|c|c|}
\hline & \multicolumn{2}{|c|}{ Fat mass [kg] } & \multicolumn{2}{|c|}{ Android fat mass [kg] } & \multicolumn{2}{|c|}{ Waist circumference $[\mathrm{cm}]$} & \multicolumn{2}{|c|}{ Waist-to-hip ratio $\times 100$} \\
\hline & B & $95 \% \mathrm{Cl}$ & B & $95 \% \mathrm{Cl}$ & B & $95 \% \mathrm{Cl}$ & B & $95 \% \mathrm{Cl}$ \\
\hline Intercept (PRE-POST) & $26.66^{* * *}$ & {$[24.59,28.73]$} & $2.46^{* * *}$ & {$[2.19,2.73]$} & $85.41^{* * *}$ & {$[82.81,88.00]$} & $84.95^{* * *}$ & {$[82.85,87.04]$} \\
\hline \multicolumn{9}{|l|}{ Group } \\
\hline PRE-POST (ref.) & - & & - & & - & & - & \\
\hline PRE-PRE & 0.46 & {$[-2.33,3.26]$} & 0.04 & {$[-0.27,0.35]$} & 1.96 & {$[-1.30,5.22]$} & 1.10 & {$[-0.92,3.12]$} \\
\hline ACC-MAD [10 mg] & $-0.77^{* *}$ & {$[-1.27,-0.26]$} & $-0.11^{* *}$ & {$[-0.18,-0.03]$} & $-0.92^{* *}$ & {$[-1.60,-0.24]$} & $-0.89^{* *}$ & {$[-1.51,-0.27]$} \\
\hline Age at baseline [year] & 0.40 & {$[-0.18,0.98]$} & 0.04 & {$[-0.02,0.11]$} & 0.61 & {$[-0.07,1.29]$} & 0.32 & {$[-0.08,0.72]$} \\
\hline \multicolumn{9}{|l|}{$\begin{array}{l}\text { Use of hormonal } \\
\text { preparations }\end{array}$} \\
\hline Non-user (ref.) & - & & - & & - & & - & \\
\hline \multicolumn{9}{|l|}{ Interactions } \\
\hline \multicolumn{9}{|l|}{ Time $\times$ Group } \\
\hline Time $\times$ PRE-POST (ref.) & - & & - & & - & & - & \\
\hline Time $\times$ PRE-PRE & $-1.33^{*}$ & {$[-2.41,-0.26]$} & $-0.15^{*}$ & {$[-0.29,-0.02]$} & -0.19 & {$[-1.61,1.22]$} & 0.61 & {$[-0.81,2.03]$} \\
\hline Time $\times$ POST-POST & $-1.20^{*}$ & {$[-2.12,-0.28]$} & -0.12 & {$[-0.24,0.00]$} & -0.47 & {$[-1.76,0.81]$} & 0.36 & {$[-0.92,1.64]$} \\
\hline Time $\times$ ACC-MAD & 0.24 & {$[-0.28,0.75]$} & 0.03 & {$[-0.04,0.09]$} & 0.23 & {$[-0.46,0.91]$} & $0.72^{*}$ & {$[0.05,1.38]$} \\
\hline
\end{tabular}

CI Confidence interval, PRE-POST participants who were pre- or perimenopausal at baseline and postmenopausal at follow-up (reference group), PRE-PRE participants who were pre- or perimenopausal in both measurements, POST-POST participants who were postmenopausal already at baseline, ACC-MAD accelerometer-measured mean amplitude deviation, $m g$ milligravity $\left(0.00981 \mathrm{~m} / \mathrm{s}^{2}\right)$, Time from baseline to follow-up.

${ }^{*} p \leq 0.05 ;{ }^{* *} p \leq 0.01 ;{ }^{* * *} p<0.001$.

\section{Body composition and anthropometrics}

ACC-MAD was inversely associated with total fat mass $(B=-0.77$, $95 \% \mathrm{Cl}[-1.27,-0.26])$ and android fat mass $(B=-0.12,95 \% \mathrm{Cl}$ $[-0.18,-0.03])$, waist circumference $(B=-0.92,95 \% \mathrm{Cl}[-1.60$, $-0.24])$, and WHR $(B=-0.89,95 \% \mathrm{Cl}[-1.51,-0.27])$ in the full sample (Table 3$)$. Total $(B=1.72,95 \% \mathrm{Cl}[0.16,3.28])$ and android fat mass $(B=0.26,95 \% \mathrm{Cl}[0.06,0.46])$ increased during the followup in the PRE-POST group and the change was smaller in the PREPRE and POST-POST groups compared to the PRE-POST group. The change in ACC-MAD was directly associated with the change in WHR ( $B=0.72,95 \% \mathrm{Cl}[0.05,1.38])$. Combined progestogen and estrogen use was associated with lower android fat mass when compared to non-hormone users. The results were relatively similar when using SR-PA, however, SR-PA was not associated with the change in WHR (Supplementary Table 4).

\section{Blood pressure}

ACC-MAD was not associated with SBP and DBP in the full sample (Table 4). SBP increased during the follow-up in the PRE-POST group $(B=9.37,95 \% \mathrm{Cl}[3.34,15.39])$ and the change did not differ between the groups. Additionally, the change in ACC-MAD was inversely associated with the change in SBP $(B=-2.40,95 \% \mathrm{Cl}$ $[-4.34,-0.46])$, but this association was not observed with SR-PA (Supplementary Table 5). The combined progestogen and estrogen use was associated with lower SBP and DBP. The results did not differ notably when excluding participants using antihypertensives (Supplementary Table 6).

\section{Number of MetS risk factors}

In the Poisson mixed-effect models (Table 5), age at baseline was directly associated with the number of MetS risk factors at baseline $(\exp (B)=1.07,95 \% \mathrm{Cl}[1.00,1.14])$ in the full sample. The number of risk factors at baseline and the change in the number of risk factors during the follow-up did not differ between the groups. Furthermore, ACC-MAD was not associated with the number of risk factors at baseline nor with the change in the number. The results did not differ notably when using SR-PA (Supplementary Table 7) or excluding participants using lipidmodifying agents or antihypertensives (Supplementary Table 8).

\section{DISCUSSION}

In this longitudinal study of middle-aged women, an increase in several indicators of metabolic health, ranging from blood-based biomarkers and SBP to body adiposity, were observed during the follow-up. The increase was greater during menopausal transition, and the rate of change decelerated after menopause, especially in blood-based biomarkers. Higher PA was associated with favorable levels in metabolic health indicators; however, the change in PA did not associate with the rate of change during the follow-up in most of the studied metabolic health indicators. Nonetheless, associations of higher PA with a greater increase in WHR and a smaller increase in SBP were observed. PA was not associated with the number of MetS risk factors.

We observed a significant increase in total cholesterol, HDL-C, LDL-C, triglycerides, and blood glucose in women going through menopause during the follow-up. Several other longitudinal studies have also reported an increase in serum total cholesterol, LDL-C, and triglycerides during the menopausal transition [37-40]. However, the literature on the associations of menopause and $\mathrm{HDL}-\mathrm{C}$ is more inconsistent. Previous studies have reported HDL-C to increase $[12,19,39,41]$, peak right before menopause [40], as 
Table 4. Pooled fixed effect estimates for blood pressure $(n=298)$.

\section{Systolic blood pressure [mmHg]}

Intercept (PRE-POST)

Main effects

Group

PRE-POST (ref.)

PRE-PRE

POST-POST

ACC-MAD [10 mg]

Age at baseline [year]

Use of hormonal preparations

Non-user (ref.)

Progestogen

Estrogen

Progestogen + Estrogen

Time (PRE-POST)

Interactions

Time $\times$ Group

Time $\times$ PRE-POST (ref.)

Time $\times$ PRE-PRE

Time $\times$ POST-POST

Time $\times$ ACC-MAD
95\% Cl

[125.01, 136.80]

\section{Diastolic blood pressure} [mmHg]

B

$84.90^{* * *}$

95\% Cl

$[81.83,87.96]$

$\mathrm{Cl}$ Confidence interval, PRE-POST participants who were pre- or perimenopausal at baseline and postmenopausal at follow-up (reference group), $P R E-P R E$ participants who were pre- or perimenopausal in both measurements, POST-POST participants who were postmenopausal already at baseline, $A C C-M A D$ accelerometer-measured mean amplitude deviation, $m g$ milligravity $\left(0.00981 \mathrm{~m} / \mathrm{s}^{2}\right)$, Time from baseline to follow-up.

${ }^{*} p \leq 0.05 ;{ }^{* *} p \leq 0.01,{ }^{* * *} p<0.001$.

well as continuously decline during menopausal transition [42]. In addition to increase in HDL-C in the PRE-POST group, higher baseline HDL-C levels and lower increase rate in the postmenopausal group were also observed. These conflicting results suggest that the change in HDL-C during menopausal transition is a complicated process related to, e.g., aging and genetic background. As HDL-C and its antiatherogenic functionality have a major role in promoting cardiovascular health, it is obvious that more detailed longitudinal studies are needed to clarify this process.

Previous findings on associations of menopausal transition and blood glucose are also contradictory. Some longitudinal studies have reported a decrease $[15,19]$ during the menopausal transition, but in cross-sectional design postmenopausal women have been reported to have higher blood glucose compared to pre- and perimenopausal women [10,43]. We observed an increase in fasting blood glucose in women going through menopause and the increase was attenuated in the POST-POST group. Our findings indicate that in addition to aging, the increase in blood glucose may be explained by the decreasing E2 levels during menopausal transition, since E2 is known to enhance insulin sensitivity and glucose disposal in women [44].

The observed increase in total and android fat masses in this study are consistent with previous literature [45-47]. The decrease in female sex hormone levels during menopausal transition is proposed to lead to increased accumulation of adipose tissue especially in the waist and visceral area $[11,48]$, yet the association of menopause to total adipose tissue accumulation is somewhat debated [9]. Although android fat mass increased during the follow-up, we did not observe a change in waist circumference. Similar results have also been reported by others $[19,49]$. This indicates a change in the ratio between android lean and fat masses during the follow-up. A comparable change in muscle-tofat ratio is also observed in total body level during the menopausal transition [11, 47]. Furthermore, we observed an increase in SBP that did not differ between the groups. This finding is supported by the previous review by Taddei [50] that suggested the changes in SBP to be more dependent on age than menopausal status in middle-aged women.

Regular PA is a well-established contributor to a healthier blood lipid profile and body composition also in menopausal women [12, 51]. With both accelerometry-measured and selfreported measures, higher PA was associated with lower levels in blood-based biomarkers and body composition variables but, surprisingly [52], not in blood pressure. When exploring the combined effect of PA and follow-up time, increased PA was associated with an accelerated increase in WHR. This result suggests accelerated decrease in hip circumference in more active women, since the change in PA was not associated with the change in waist circumference. While estradiol levels are associated with both gluteal adipose [53] and muscle mass $[14,54]$, we suspect that the pronounced decrease in more physically active women is caused especially by the loss of muscle mass due to the potentially higher muscle mass on their gluteal area at baseline. However, in the current study, we were not able to accurately identify the lost tissue type at the hip area. We also observed higher PA to be associated with a smaller increase in SBP during the follow-up. As discussed earlier, the observed changes in SBP may have been related to aging rather than menopausal transition [50], but our results indicate that regular PA may be efficient for controlling SBP in menopausal women similar to other populations $[55,56]$. 
Table 5. Pooled fixed effect estimates for number of metabolic syndrome risk factors $(n=298)$.

\begin{tabular}{|c|c|c|}
\hline & \multicolumn{2}{|c|}{$\begin{array}{l}\text { Number of metabolic } \\
\text { syndrome risk factors }\end{array}$} \\
\hline & $\exp (B)$ & $95 \% \mathrm{Cl}$ \\
\hline Intercept (PRE-POST) & 1.41 & {$[0.91,2.16]$} \\
\hline \multicolumn{3}{|l|}{ Main effects } \\
\hline \multicolumn{3}{|l|}{ Group } \\
\hline PRE-POST (ref.) & - & \\
\hline PRE-PRE & 1.31 & {$[0.93,1.84]$} \\
\hline POST-POST & 0.95 & {$[0.71,1.28]$} \\
\hline ACC-MAD [10 mg] & 0.91 & {$[0.79,1.04]$} \\
\hline Age at baseline [year] & $1.07^{*}$ & {$[1.00,1.14]$} \\
\hline \multicolumn{3}{|c|}{ Use of hormonal preparations } \\
\hline Non-user (ref.) & - & \\
\hline Progestogen & 0.98 & {$[0.78,1.23]$} \\
\hline Estrogen & 0.85 & {$[0.41,1.74]$} \\
\hline Progestogen + Estrogen & 0.71 & {$[0.50,1.02]$} \\
\hline Time (PRE-POST) & 1.59 & {$[0.93,2.73]$} \\
\hline \multicolumn{3}{|l|}{ Interactions } \\
\hline \multicolumn{3}{|l|}{ Time $\times$ Group } \\
\hline Time $\times$ PRE-POST (ref.) & - & \\
\hline Time $\times$ PRE-PRE & 0.77 & {$[0.53,1.14]$} \\
\hline Time $\times$ POST-POST & 0.93 & {$[0.67,1.30]$} \\
\hline Time $\times$ ACC-MAD & 0.88 & {$[0.73,1.06]$} \\
\hline
\end{tabular}

$\mathrm{Cl}$ Confidence interval, PRE-POST participants who were pre- or perimenopausal at baseline and postmenopausal at follow-up (reference group), PRE-PRE participants who were pre- or perimenopausal in both measurements, POST-POST participants who were postmenopausal already at baseline, ACC-MAD accelerometer-measured mean amplitude deviation, $\mathrm{mg}$ milligravity $\left(0.00981 \mathrm{~m} / \mathrm{s}^{2}\right)$, Time from baseline to follow-up.

${ }^{*} p \leq 0.05 ;{ }^{* *} p \leq 0.01 ;{ }^{* *} p<0.001$.

Although PA was associated with individual indicators of metabolic health, no associations with PA and the number of MetS risk factors or change in the number were observed. This may be caused by the strict cutoff points used in the clinical identification of MetS that does not capture the change unless the cutoff point is reached. Our findings are somewhat contradictory to a recent longitudinal study [57] in which higher PA was associated with lower incidence and better recovery from MetS in middle-aged women. Nonetheless, also in our study, the number of MetS factors tended to be smaller and the increase in the number was slightly lower in more active participants. Thus, PA might be beneficial for preventing the unwanted changes in individual MetS risk factors, but more studies on the associations of PA and number of MetS risk factors during menopause are required.

An interesting additional finding of the study was the observed associations of external hormone use with multiple indicators of metabolic health, highlighted by the distinctive association between the combined use of estrogen and progestogen and lower SBP. The use of hormone replacement therapy has been previously shown to reduce abdominal fat, blood glucose, LDL-toHDL ratio and blood pressure [58], similar to our results. The individual effects of progestogen use on body composition and metabolic health are less studied, but estrogen is recognized to associate directly with gynoid adipose tissue volume [48, 59], better insulin sensitivity [44], and beneficial effects on vasodilatation and LDL-C concentration [60]. Although our results from exogenous hormone use are mostly in agreement with previous results, the results need to be interpreted with caution, since we did not consider the dosage, the duration of use, or form of the exogenous hormones.

One of the limitations was that the measurements were repeated only once. The homogenous sample of white, middleaged women with exclusion of women with severe obesity and different medical disorders may limit the generalizability of the results for more heterogeneous populations including participants with disabling conditions. Furthermore, based on the sensitivity analysis, dropouts during the study have caused healthy selection bias particularly towards slightly better glucose control and higher PA which also limits the generalizability of the results. However, this is unlikely to have caused overestimation of the observed unhealthy menopause-related changes in outcome variables. The strengths of the study included the use of accelerometers for PA and DXA for body composition measurements. Additionally, the study design in which women of similar age but different menopausal status were followed for the same amount of time allowed to study the menopause-related changes in outcome variables while taking into account the simultaneous aging.

In conclusion, the results indicate that undesirable changes in blood lipids, body adiposity, and blood pressure occur in middleaged women, and the rate of change accelerates near menopause, especially in blood lipids. Although habitual PA associated with a healthier blood lipid profile and lower body adiposity in middleaged women in this study, it did not significantly modulate the menopause-related changes in most of the studied metabolic health indicators. However, higher PA may attenuate the increase in SBP and associate with an accelerated increase in WHR. These results indicate that significant increases in PA around menopause may be needed to counteract the menopause-related changes in blood-based biomarkers and body adiposity. Nonetheless, our findings could encourage professionals working with menopausal women to highlight the importance of PA in the early prevention of hypertension and cardiovascular disease. Further longitudinal studies on the role of PA on the metabolic health during the menopausal transition are needed.

\section{REFERENCES}

1. Grundy SM, Hansen B, Smith SC Jr, Cleeman Jl, Kahn RA. Clinical Management of Metabolic Syndrome: Report of the American Heart Association/National Heart, Lung, and Blood Institute/American Diabetes Association Conference on Scientific Issues Related to Management. Circulation. 2004;109:551-6. https://doi.org/ 10.1161/01.CIR.0000112379.88385.67.

2. Malik S, Wong ND, Franklin SS, Kamath TV, L'Italien GJ, Pio JR, et al. Impact of the metabolic syndrome on mortality from coronary heart disease, cardiovascular disease, and all causes in United States adults. Circulation. 2004;110:1245-50. https://doi.org/10.1161/01.CIR.0000140677.20606.0E.

3. Ford ES, Li C, Sattar N. Metabolic syndrome and incident diabetes: current state of the evidence. Diabetes Care. 2008;31:1898-904. https://doi.org/10.2337/dc080423.

4. Poulsen P, Vaag A, Kyvik K, Beck-Nielsen H. Genetic versus environmental aetiology of the metabolic syndrome among male and female twins. Diabetologia. 2001;44:537-43. https://doi.org/10.1007/s001250051659.

5. Gold EB. The timing of the age at which natural menopause occurs. Obstet Gynecol Clin North Am. 2011;38:425-40. https://doi.org/10.1016/j. ogc.2011.05.002.

6. Dratva J, Gómez Real F, Schindler C, Ackermann-Liebrich U, Gerbase MW, ProbstHensch NM, et al. Is age at menopause increasing across Europe? Results on age at menopause and determinants from two population-based studies. Menopause. 2009;16:385-94. https://doi.org/10.1097/gme.0b013e31818aefef.

7. Kontis V, Bennett JE, Mathers CD, Li G, Foreman K, Ezzati M. Future life expectancy in 35 industrialised countries: projections with a Bayesian model ensemble. Lancet. 2017;389:1323-35. https://doi.org/10.1016/S0140-6736(16)32381-9.

8. Carr MC. The emergence of the metabolic syndrome with menopause. J Clin Endocrinol Metab. 2003;88:2404-11. https://doi.org/10.1210/jc.2003-030242.

9. El Khoudary SR, Aggarwal B, Beckie TM, Hodis HN, Johnson AE, Langer RD, et al. Menopause transition and cardiovascular disease risk: implications for timing of early prevention: a scientific statement from the American Heart Association. Circulation. 2020;142:e506-32. https://doi.org/10.1161/CIR.0000000000000912. 
10. Otsuki M, Kasayama S, Morita S, Asanuma N, Saito H, Mukai M, et al. Menopause, but not age, is an independent risk factor for fasting plasma glucose levels in nondiabetic women. Menopause. 2007;14:404-7. https://doi.org/10.1097/01. gme.0000247014.56254.12.

11. Ambikairajah A, Walsh $E$, Tabatabaei-Jafari $H$, Cherbuin N. Fat mass changes during menopause: a metaanalysis. Am J Obstet Gynecol. 2019;221:393-409.e50. https://doi.org/10.1016/j.ajog.2019.04.023.

12. Karvinen $S$, Jergenson MJ, Hyvärinen $M$, Aukee $P$, Tammelin $T$, Sipilä $S$, et al. Menopausal status and physical activity are independently associated with cardiovascular risk factors of healthy middle-aged women: cross-sectional and longitudinal evidence. Front Endocrinol. 2019;10. https://doi.org/10.3389/ fendo.2019.00589.

13. Wang Q, Ferreira DLS, Nelson SM, Sattar N, Ala-Korpela M, Lawlor DA. Metabolic characterization of menopause: cross-sectional and longitudinal evidence. BMC Med. 2018;16:17. https://doi.org/10.1186/s12916-018-1008-8.

14. Juppi H-K, Sipilä S, Cronin NJ, Karvinen S, Karppinen JE, Tammelin TH, et al. Role of menopausal transition and physical activity in loss of lean and muscle mass: a follow-up study in middle-aged Finnish Women. J Clin Med. 2020;9. https://doi. org/10.3390/jcm9051588.

15. Janssen I, Powell LH, Crawford S, Lasley B, Sutton-Tyrrell K. Menopause and the metabolic syndrome: The Study of Women's Health Across the Nation. Arch Intern Med. 2008;168:1568-75. https://doi.org/10.1001/archinte.168.14.1568.

16. Warburton DER, Nicol CW, Bredin SSD. Health benefits of physical activity: the evidence. CMAJ. 2006;174:801-9. https://doi.org/10.1503/cmaj.051351.

17. Lakka TA, Laaksonen DE. Physical activity in prevention and treatment of the metabolic syndrome. Appl Physiol Nutr Metab. 2007;32:76-88. https://doi.org/ 10.1139/h06-113.

18. Wewege MA, Thom JM, Rye K, Parmenter BJ. Aerobic, resistance or combined training: a systematic review and meta-analysis of exercise to reduce cardiovascular risk in adults with metabolic syndrome. Atherosclerosis. 2018;274:162-71. https://doi.org/10.1016/j.atherosclerosis.2018.05.002.

19. Abdulnour J, Doucet É, Brochu M, Lavoie J, Strychar I, Rabasa-Lhoret R, et al. The effect of the menopausal transition on body composition and cardiometabolic risk factors: a Montreal-Ottawa New Emerging Team group study. Menopause. 2012;19:760-7. https://doi.org/10.1097/gme.0b013e318240f6f3.

20. Kovanen V, Aukee P, Kokko K, Finni T, Tarkka IM, Tammelin T, et al. Design and protocol of Estrogenic Regulation of Muscle Apoptosis (ERMA) study with 47 to 55-year-old women's cohort: novel results show menopause-related differences in blood count. Menopause. 2018;25:1020-32. https://doi.org/10.1097/ GME.0000000000001117.

21. Snijder MB, Dekker JM, Visser M, Bouter LM, Stehouwer CD, Kostense PJ, et al. Associations of hip and thigh circumferences independent of waist circumference with the incidence of type 2 diabetes: the Hoorn Study. Am J Clin Nutr. 2003;77:1192-7. https://doi.org/10.1093/ajcn/77.5.1192.

22. Vähä-Ypyä $H$, Vasankari $T$, Husu $P$, Suni J, Sievänen $H$. A universal, accurate intensity-based classification of different physical activities using raw data of accelerometer. Clin Physiol Funct Imaging. 2015;35:64-70. https://doi.org/ 10.1111/cpf.12127.

23. Rowlands AV. Moving forward with accelerometer-assessed physical activity: two strategies to ensure meaningful, interpretable, and comparable measures. Pediatr Exerc Sci. 2018;30:450-6. https://doi.org/10.1123/pes.2018-0201.

24. Vähä-Ypyä H, Vasankari T, Husu $P$, Mänttäri A, Vuorimaa T, Suni J, et al. Validation of cut-points for evaluating the intensity of physical activity with accelerometrybased mean amplitude deviation (MAD). Plos ONE. 2015;10:e0134813. https://doi. org/10.1371/journal.pone.0134813.

25. Hyvärinen M, Sipilä S, Kulmala J, Hakonen H, Tammelin TH, Kujala UM, et al. Validity and reliability of a single question for leisure-time physical activity assessment in middle-aged women. J Aging Phys Act. 2019;28:231-41. https:// doi.org/10.1123/japa.2019-0093.

26. Kujala UM, Kaprio J, Sarna S, Koskenvuo M. Relationship of leisure-time physical activity and mortality: the Finnish twin cohort. JAMA. 1998;279:440-4. https://doi. org/10.1001/jama.279.6.440.

27. The WHO Collaborating Centre for Drug Statistics Methodology. ATC: structure and principles. 2018. https://www.whocc.no/atc/structure_and_principles/. Accessed 29 April 2021

28. Masip G, Keski-Rahkonen A, Pietiläinen KH, Kujala UM, Rottensteiner M, Väisänen $\mathrm{K}$, et al. Development of a Food-Based Diet Quality Score from a Short FFQ and Associations with Obesity Measures, Eating Styles and Nutrient Intakes in Finnish Twins. Nutrients. 2019;11. https://doi.org/10.3390/nu11112561.

29. $\mathrm{R}$ Core Team. R: a language and environment for statistical computing. Version 4.0.5. Vienna, Austria: R Foundation for Statistical Computing; 2021. https://www. R-project.org/.

30. van Buuren $S$, Groothuis-Oudshoorn K. Mice: multivariate imputation by chained equations in R. J Stat Softw. 2011;45:1-67. https://doi.org/10.18637/jss.v045.i03.
31. Rubin DB. Multiple imputation for nonresponse in surveys. New Jersey, USA: John Wiley \& Sons; 2004.

32. Laird NM, Ware JH. Random-effects models for longitudinal data. Biometrics. 1982;38:963-74. https://doi.org/10.2307/2529876.

33. Pinheiro J, Bates D, DebRoy S, Sarkar D, R Core Team. nmle: linear and nonlinear mixed effects models. $\mathrm{R}$ package version 3.1-152. 2021. https://cran.r-project.org/ web/packages/nlme/index.html.

34. Bates $D$, Mächler $M$, Bolker $B$, Walker $S$. Fitting linear mixed-effects models using Ime4. J Stat Softw. 2015;67:1-48. https://doi.org/10.18637/jss.v067.i01.

35. WHO. Prevention of cardiovascular disease: guidelines for assessment and management of total cardiovascular risk. World Health Organization; 2007. https://apps.who.int/iris/handle/10665/43685.

36. Williams B, Mancia G, Spiering W, Agabiti Rosei E, Azizi M, Burnier M, et al. 2018 ESC/ESH Guidelines for the management of arterial hypertension: The Task Force for the management of arterial hypertension of the European Society of Cardiology (ESC) and the European Society of Hypertension (ESH). Eur Heart J. 2018;39:3021-104. https://doi.org/10.1093/eurheartj/ehy339.

37. Jensen J, Nilas L, Christiansen C. Influence of menopause on serum lipids and lipoproteins. Maturitas. 1990;12:321-31. https://doi.org/10.1016/0378-5122(90) 90012-U.

38. Stevenson JC, Crook D, Godsland IF. Influence of age and menopause on serum lipids and lipoproteins in healthy women. Atherosclerosis. 1993;98:83-90. https:// doi.org/10.1016/0021-9150(93)90225-j.

39. Derby CA, Crawford SL, Pasternak RC, Sowers M, Sternfeld B, Matthews KA. Lipid changes during the menopause transition in relation to age and weight: the Study of Women's Health Across the Nation. Am J Epidemiol. 2009;169:1352-61. https://doi.org/10.1093/aje/kwp043.

40. Matthews KA, Crawford SL, Chae CU, Everson-Rose SA, Sowers MF, Sternfeld B, et al. Are changes in cardiovascular disease risk factors in midlife women due to chronological aging or to the menopausal transition? J Am Coll Cardiol. 2009;54:2366-73. https://doi.org/10.1016/j.jacc.2009.10.009.

41. Do K-A, Green A, Guthrie JR, Dudley EC, Burger HG, Dennerstein L. Longitudinal study of risk factors for coronary heart disease across the menopausal transition. Am J Epidemiol. 2000;151:584-93. https://doi.org/10.1093/oxfordjournals.aje. a010246.

42. Gurka MJ, Vishnu A, Santen RJ, DeBoer MD. Progression of metabolic syndrome severity during the menopausal transition. J Am Heart Assoc. 2016;5. https://doi. org/10.1161/JAHA.116.003609.

43. Lynch NA, Ryan AS, Berman DM, Sorkin JD, Nicklas BJ. Comparison of VO2max and disease risk factors between perimenopausal and postmenopausal women Menopause. 2002;9:456-62. https://doi.org/10.1097/00042192-200211000-00012.

44. Mauvais-Jarvis F, Clegg DJ, Hevener AL. The role of estrogens in control of energy balance and glucose homeostasis. Endocr Rev. 2013;34:309-38. https://doi.org/ 10.1210/er.2012-1055.

45. Ho SC, Wu S, Chan SG, Sham A. Menopausal transition and changes of body composition: a prospective study in Chinese perimenopausal women. Int J Obes. 2010;34:1265-74. https://doi.org/10.1038/ijo.2010.33.

46. Razmjou S, Abdulnour J, Bastard J, Fellahi S, Doucet É, Brochu M, et al. Body composition, cardiometabolic risk factors, physical activity, and inflammatory markers in premenopausal women after a 10-year follow-up: a MONET study. Menopause. 2018;25:89-97. https://doi.org/10.1097/GME.0000000000000951.

47. Greendale GA, Sternfeld B, Huang M, Han W, Karvonen-Gutierrez C, Ruppert K, et al. Changes in body composition and weight during the menopause transition. JCI Insight. 2019;4. https://doi.org/10.1172/jci.insight.124865.

48. Wang $\mathrm{H}$, Eckel RH. Lipoprotein lipase: from gene to obesity. Am J Physiol Endocrinol Metab. 2009;297:271-88. https://doi.org/10.1152/ajpendo.90920.2008.

49. Franklin RM, Ploutz-Snyder L, Kanaley JA. Longitudinal changes in abdominal fat distribution with menopause. Metabolism. 2009;58:311-5. https://doi.org/ 10.1016/j.metabol.2008.09.030.

50. Taddei S. Blood pressure through aging and menopause. Climateric. 2009;12:36-40. https://doi.org/10.1080/13697130903004758.

51. Sternfeld B, Bhat AK, Wang H, Sharp T, Quesenberry CP. Menopause, physical activity, and body composition/fat distribution in midlife women. Med Sci Sports Exerc. 2005;37:1195-202. https://doi.org/10.1249/01.mss.0000170083.41186.b1.

52. Cornelissen VA, Fagard RH. Effects of endurance training on blood pressure, blood pressure-regulating mechanisms, and cardiovascular risk factors. Hypertension. 2005;46:667-75. https://doi.org/10.1161/01.HYP.0000184225.05629.51.

53. Pedersen SB, Kristensen $K$, Hermann PA, Katzenellenbogen JA, Richelsen $B$. Estrogen controls lipolysis by up-regulating a2A-adrenergic receptors directly in human adipose tissue through the estrogen receptor $a$. implications for the female fat distribution. J Clin Endocrinol Metab. 2004;89:1869-78. https://doi.org/ 10.1210/jc.2003-031327

54. Sipilä S, Törmäkangas T, Sillanpää E, Aukee $P$, Kujala UM, Kovanen V, et al. Muscle and bone mass in middle-aged women: role of menopausal status and physical 
activity. J Cachexia Sarcopenia Muscle. 2020;11:678-709. https://doi.org/10.1002/ jcsm.12547.

55. Hu G, Barengo NC, Tuomilehto J, Lakka TA, Nissinen A, Jousilahti P. Relationship of physical activity and body mass index to the risk of hypertension: a prospective study in Finland. Hypertension. 2004;43:25-30. https://doi.org/10.1161/ 01.HYP.0000107400.72456.19.

56. Börjesson M, Onerup A, Lundqvist S, Dahlöf B. Physical activity and exercise lower blood pressure in individuals with hypertension: narrative review of $27 \mathrm{RCTs}$. Br J Sports Med. 2016;50:356-61. https://doi.org/10.1136/bjsports-2015-095786.

57. Ward E, Gold EB, Johnson WO, Ding F, Chang P, Song P, et al. Patterns of cardiometabolic health as midlife women transition to menopause: a prospective multiethnic study. J Clin Endocrinol Metab. 2019;104:1404-12. https://doi.org/ 10.1210/jc.2018-00941.

58. Salpeter SR, Walsh JME, Ormiston TM, Greyber E, Buckley NS, Salpeter EE. Metaanalysis: effect of hormone-replacement therapy on components of the metabolic syndrome in postmenopausal women. Diabetes Obes Metab. 2006;8:538-54. https://doi.org/10.1111/j.1463-1326.2005.00545.x.

59. Lindberg UB, Crona N, Silfverstolpe G, Björntorp P, Rebuffé-Scrive M. Regional adipose tissue metabolism in postmenopausal women after treatment with exogenous sex steroids. Horm Metab Res. 1990;22:345-51. https://doi.org/ 10.1055/s-2007-1004917.

60. Dubey RK, Jackson EK. Estrogen-induced cardiorenal protection: potential cellular, biochemical, and molecular mechanisms. Am J Physiol Renal Physiol. 2001;280. https://doi.org/10.1152/ajprenal.2001.280.3.F365.

\section{ACKNOWLEDGEMENTS}

The authors would like to thank Anja Ahlgren, Eeva-Maija Palonen, Mervi Matero, Hanne Tähti, and the other laboratory staff in Faculty of Sport and Health Sciences for their invaluable help with the data collection, as well as the participants in ERMA and EsmiRs studies who volunteered their time and effort.

\section{AUTHOR CONTRIBUTIONS}

$\mathrm{MH}$ and HKJ prepared the original draft and share the first authorship of the paper. $\mathrm{MH}$ was the major contributor in statistical analyses, while ST offered guidance. MH analyzed the raw accelerometer data and HKJ processed the DXA data. JEK was responsible for forming the diet analysis used. VK and EKL obtained funding for the project. ST, JEK, SK, THT, VK, PA, UMK, TR, SS, and EKL all gave their professional effort in the writing process. All authors read and approved the final paper.

\section{FUNDING}

The study was supported by the Academy of Finland (grant numbers 275323 to VK and 309504, 314181 and 335249 to EKL). TR was an Academy Research Fellow during the preparation of this paper (Academy of Finland grant numbers 321336 and 328818). Open Access funding provided by University of Jyväskylä (JYU).

\section{COMPETING INTERESTS}

The authors declare no competing interests.

\section{ADDITIONAL INFORMATION}

Supplementary information The online version contains supplementary material available at https://doi.org/10.1038/s41366-021-01022-x.

Correspondence and requests for materials should be addressed to Matti Hyvärinen.

Reprints and permission information is available at http://www.nature.com/ reprints

Publisher's note Springer Nature remains neutral with regard to jurisdictional claims in published maps and institutional affiliations.

\section{(c) (i)}

Open Access This article is licensed under a Creative Commons Attribution 4.0 International License, which permits use, sharing, adaptation, distribution and reproduction in any medium or format, as long as you give appropriate credit to the original author(s) and the source, provide a link to the Creative Commons license, and indicate if changes were made. The images or other third party material in this article are included in the article's Creative Commons license, unless indicated otherwise in a credit line to the material. If material is not included in the article's Creative Commons license and your intended use is not permitted by statutory regulation or exceeds the permitted use, you will need to obtain permission directly from the copyright holder. To view a copy of this license, visit http://creativecommons. org/licenses/by/4.0/.

(c) The Author(s) 2021 